\title{
Lower Bounds for 1-, 2- and 3-Dimensional On-Line Bin Packing Algorithms
}

\author{
G. Galambos*, Szeged, and A. van Vliet ${ }^{\dagger}$, Rotterdam
}

Received December 27, 1993

\begin{abstract}
Zusammenfassung
Lower Bounds for 1-, 2- and 3-Dimensional On-Line Bin Packing Algorithms. In this paper we discuss lower bounds for the asymptotic worst case ratio of on-line algorithms for different kind of bin packing problems. Recently, Galambos and Frenk gave a simple proof of the $1.536 \ldots$ lower bound for the 1-dimensional bin packing problem. Following their ideas, we present a general technique that can be used to derive lower bounds for other bin packing problems as well. We apply this technique to prove new lower bounds for the 2-dimensional $(1.802 \ldots)$ and 3-dimensional $(1.974 \ldots)$ bin packing problem.
\end{abstract}

AMS Subject Classifications: 90B35, 90C27

Key words: Combinatorial problems, on-line, bin packing, suboptimal algorithms.

Untere Schranken für. 1-, 2- und 3-dimensionale Bin-Packungsprobleme. In dieser Arbeit untersuchen wir asymptotische untere Schranken von on-line Algorithmen für verschiedene Arten des BinPackungsproblems. Kürzlich haben Galambos und Frenk einen einfachen Beweis der unteren Schranke 1.536 ... für das eindimensionale Packungsproblem angegeben. Ausgehend von ihren Überlegungen präsentieren wir eine allgemeine Technik zur Herleitung unterer Schranken auch für andere Packungsprobleme. Wir verwenden diese Technik, um neue untere Schranken für das zweidimensionale $(1,802 \ldots)$ und das dreidimensionale $(1,974 \ldots)$ Packungsproblem zu beweisen.

\section{Introduction}

The 1-dimensional bin packing problem can be stated as follows. We are given a list $L=\left(a_{1}, a_{2}, \ldots, a_{n}\right)$ of items. An item $a_{i}$ has a size $s\left(a_{i}\right)$ that satisfies $0<s\left(a_{i}\right) \leq 1$. Further we have an infinite supply of unit-capacity bins. The objective is to pack the items in a minimum number of bins such that the total size of the items in every bin is less than or equal to 1 .

In the 2-dimensional bin packing problem the items are rectangles with width $0<w\left(a_{i}\right) \leq 1$ and height $0<h\left(a_{i}\right) \leq 1$ that have to be packed in a minimum number of unit-square bins such that

* Department of Computer Science, JGYTF, P.O. Box 396, H-6720 Szeged, Hungary.

† Econometric Institute, Erasmus University Rotterdam, P.O. Box 1738, 3000 DR Rotterdam, The Netherlands. 
- each item is contained entirely within its bin with the sides of the item parallel to the sides of the bin,

- no two items overlap.

Moreover, the items have a fixed orientation, so that rotation is not allowed.

Finally, we have the 3-dimensional bin packing problem where the items are boxes with width $0<w\left(a_{i}\right) \leq 1$, height $0<h\left(a_{i}\right) \leq 1$ and depth $0<d\left(a_{i}\right) \leq 1$ that have to be packed in a minimum number of unit-cubes under the same conditions as in its 2-dimensional variant.

Since these bin packing problems have been proved to be NP-hard (see [5]), research has focused on finding good approximate algorithms. The number of bins that an algorithm $A$ uses to pack list $L$ is denoted by $A(L)$ and the optimum (minimum) number of bins for list $L$ is denoted by $O P T(L)$. We will measure the performance of an algorithm $A$ by its asymptotic worst case ratio $R_{A}^{\infty}$, which is defined as

$$
R_{A}^{\infty}=\limsup _{k \rightarrow \infty}\left(\max _{L}\left\{\frac{A(L)}{k} \mid O P T(L)=k\right\}\right) .
$$

A special class of algorithms are the so-called on-line algorithms. An algorithm is called an on-line algorithm if it packs the items in the order given by the list, without knowledge of the subsequent items on the list. This lack of knowledge is such a severe handicap that no on-line algorithm can have an asymptotic worst case ratio close to 1 . The best on-line algorithm for the 1-dimensional problem is due to Richey [8] and yields an asymptotic worst case ratio of $1.589 \ldots$. For higher dimensions $\mathrm{Li}$ and Cheng [6] and Csirik and Van Vliet [1] gave different algorithms that both have an asymptotic worst case ratio close to $(1.691)^{d}$, where $d$ is the dimension. On the other hand, Liang [7] showed that no on-line algorithm for the 1-dimensional bin packing problem can have an asymptotic worst case ratio better than $1.536 \ldots$. Galambos and Frenk [3] gave a simplified proof for this lower bound with a technique that can easily be used to derive lower bounds for other bin packing problems as well. It was used by Galambos [2] to derive a 1.6 lower bound for the 2-dimensional bin packing problem, and by Galambos et al. [4] to derive a lower bound for the $d$-dimensional vector packing problem.

In this paper we show the basic steps of this technique and we will illustrate its application with the proof of Galambos and Frenk for the 1-dimensional bin packing problem. Furthermore, we will use it to derive new lower bounds for the 2- and 3-dimensional case. We will also give a conjecture on a lower bound for the general $d$-dimensional bin packing problem. We will conclude with a discussion of a linear programming technique due to Van Vliet [9] that can be used to slightly improve on these lower bounds. 


\section{The Technique}

In this section we will describe a general technique to derive a lower bound for the asymptotic worst case ratio of any on-line algorithm for the bin packing problem. Suppose there is a list $L$ which is a concatenation of $k+1$ sublists: $L=$ $L_{k} L_{k-1} \ldots L_{0}$. Each of these sublists contains $n$ equal sized elements $a_{j i}, 0 \leqq j \leqq n$, $1 \leq i \leq n$, whose size does not depend on $n$. An on-line algorithm $A$ has to pack this concatenated list and we evaluate its performance ratio after packing each of the sublists:

$$
r_{j}(n)=\frac{A\left(L_{k} \ldots L_{j}\right)}{O P T\left(L_{k} \ldots L_{j}\right)} .
$$

If we let

$$
r=\max _{j}\left\{\lim _{n \rightarrow \infty} r_{j}(n)\right\}
$$

then $r$ will be less than or equal to the asymptotic worst case ratio of algorithm $A$. Because we are interested in a lower bound for the asymptotic worst case ratio of any on-line algorithm, we will give a lower bound for $r$ that holds for every $A$.

We now introduce the following notations.

- $\mathscr{B}=\left\{B_{1}, \ldots, B_{A\left(L_{k} \ldots L_{0}\right)}\right\}$ denotes the packing of the concatenated list $L_{k} \ldots L_{0}$ produced by the on-line heuristic $A$. A bin belonging to $\mathscr{B}$ is called a bin of type $\mathbf{t}=\left(t_{0}, t_{1}, \ldots, t_{k}\right)$ if it contains $t_{j}$ items of list $L_{j}, 0 \leq j \leq k$. Further, the number $n(\mathbf{t})$ equals the number of bins of type $\mathbf{t}$.

- The subset $\mathscr{B}_{j} \subseteq \mathscr{B}, 0 \leq j \leq k$, contains only those bins which were used for the first time by the on-line heuristic $A$ during the packing of list $L_{j}$. Moreover, define for every $0 \leq j \leq k$ the sets

$$
T_{j}=\left\{\mathbf{t} \text { : There exists a bin of type } \mathbf{t} \text { in } \mathscr{B}_{j}\right\}
$$

and

$$
T=\{\mathbf{t} \text { : There exists a bin of type } \mathbf{t} \text { in } \mathscr{B}\}
$$

(Note that if $t=\left(t_{1}, \ldots, t_{r}, \ldots, t_{k}\right)$ with $t_{i}=0 \forall i<r$ and $t_{r}>0$ then $t \in \mathscr{B}_{r}$.) These notations enable us to write

$$
A\left(L_{k} \ldots L_{j}\right)=\sum_{p=j}^{k} \sum_{\mathbf{t} \in T_{p}} n(\mathbf{t})
$$

So, now we have described the behaviour of an algorithm $A$ in a suitable way. With this we come to the main theorem:

Theorem 1. Let $w_{j}, 0 \leq j \leq k$, be some positive weights such that for every $\mathbf{t} \in T_{p}$

$$
\sum_{j=0}^{p} w_{j} t_{j} \leq p+1
$$

holds. Then 


$$
\lim _{n \rightarrow \infty} \frac{n \sum_{j=0}^{k} w_{j}}{\sum_{j=0}^{k} \operatorname{OPT}\left(L_{k} \ldots L_{j}\right)}
$$

is a lower bound for the asymptotic worst case ratio of any on-line algorithm $A$.

Proof:

From (1) we get that

$$
\sum_{j=0}^{k} A\left(L_{k} \ldots L_{j}\right)=\sum_{j=0}^{k} \sum_{p=j}^{k} \sum_{\mathbf{t} \in T_{p}} n(\mathbf{t})=\sum_{p=0}^{k}(p+1) \sum_{\mathbf{t} \in T_{p}} n(\mathbf{t}) .
$$

Further, because every sublist $L_{j}$ contains $n$ items, we have for every $0 \leq j \leq k$ that

$$
n=\sum_{\mathbf{t} \in T} t_{j} n(\mathbf{t})=\sum_{p=j}^{k} \sum_{\mathbf{t} \in T_{p}} t_{j} n(\mathbf{t}) .
$$

Multiplying this equation by $w_{j}, 0 \leq j \leq k$, and adding up, yields

$$
\begin{aligned}
n \sum_{j=0}^{k} w_{j} & =\sum_{j=0}^{k} \sum_{p=j}^{k} \sum_{\mathbf{t} \in T_{p}} w_{j} t_{j} n(\mathbf{t})=\sum_{p=0}^{k} \sum_{\mathbf{t} \in T_{p}} n(\mathbf{t}) \sum_{j=0}^{p} w_{j} t_{j} \\
& \leq \sum_{p=0}^{k}(p+1) \sum_{\mathbf{t} \in T_{p}} n(\mathbf{t})=\sum_{j=0}^{k} A\left(L_{k} \ldots L_{j}\right) .
\end{aligned}
$$

So,

$$
\begin{aligned}
r & =\max _{j}\left\{\lim _{n \rightarrow \infty} \frac{A\left(L_{k} \ldots L_{j}\right)}{\operatorname{OPT}\left(L_{k} \ldots L_{j}\right)}\right\} \\
& \geq \lim _{n \rightarrow \infty} \frac{\sum_{j=0}^{k} A\left(L_{k} \ldots L_{j}\right)}{\sum_{j=0}^{k} \operatorname{OPT}\left(L_{k} \ldots L_{j}\right)} \\
& \geq \lim _{n \rightarrow \infty} \frac{n \sum_{j=0}^{k} w_{j}}{\sum_{j=0}^{k} O P T\left(L_{k} \ldots L_{j}\right)} .
\end{aligned}
$$

Given the construction of the concatenated list $L$, the quality of the lower bound depends on the gap in inequality (2) for packings produced by an algorithm that minimizes $r$. For that reason, it is very important how we choose the weights $w_{j}$. However, as optimal algorihtms tend to have more than $k+1$ different packings, we can never guarantee to find weights that result in equality of (2) for all packings used.

We did not make any assumptions about what kind of bin packing problem we are dealing with. Hereby, this technique can be used for the 1-, 2- and 3-dimensional bin packing problem as well as for the $d$-dimensional vector packing problem to derive lower bounds for the asymptotic worst case ratio of on-line algorithms.

We summarize this technique in the following steps:

1. Construct a list $L$ consisting of several sublists $L_{j}$, that contain $n$ identical items each.

2. Choose appropriate weights $w_{j}$ such that (2) holds.

3. Give upper bounds for $O P T\left(L_{k} \ldots L_{j}\right), 0 \leq j \leq k$.

4. Calculate the lower bound according to (3). 
Note that it is sufficient to give upper bounds for $O P T\left(L_{k} \ldots L_{j}\right), 0 \leq j \leq k$, because that terms appear in the denominator of (3).

\section{1-Dimensional Bin Packing}

In this section we will use the same construction of the lists $L_{j}$ as Liang [7] to give the much simpler proof of Galambos and Frenk [3] for the $1.536 \ldots$ lower bound.

Therefore we need the series $m_{j}, j \geq 0$, which is defined by

$$
m_{0}=1 \quad \text { and } \quad m_{j}=m_{j-1}\left(m_{j+1}+1\right) \quad \forall j \geq 1 .
$$

Let $k$ be a fixed natural number. Let $L=L_{k} L_{k-1} \ldots L_{0}$ be a concatenation of $k+1$ sublists $L_{j}, 0 \leq j \leq k$, where sublist $L_{j}$ contains $n$ items of size $\frac{1}{m_{j}+1}+\varepsilon$ $\left(0<\varepsilon \leq \frac{1}{(k+1) m_{k+1}}\right)$.

In [7] it has been shown that for any partial lists $L_{k} \ldots L_{j}, 0 \leq j \leq k$, if $n$ is a multiple of $m_{k}$, then for every $0 \leq j \leq k$

$$
O P T\left(L_{k} \ldots L_{j}\right)=\frac{n}{m_{j}} .
$$

Using Theorem 1 and setting the weights $w_{j}, 0 \leq j \leq k$, such that $w_{j}=\frac{j+1}{m_{j}}$, $0 \leq j \leq k$, the desired inequality

$$
\sum_{j=0}^{p} w_{j} t_{j} \leq p+1
$$

holds for every packing $\mathbf{t}=\left(t_{0}, \ldots, t_{k}\right) \in T_{p}([3])$.

Combining everything yields the original result of Liang [7]:

Theorem 2. Every on-line algorithm for the 1-dimensional bin packing problem has an asymptotic worst case ratio of at least

$$
\lim _{k \rightarrow \infty} \frac{\sum_{j=0}^{k} \frac{j+1}{m_{j}}}{\sum_{j=0}^{k} \frac{1}{m_{j}}} .
$$

Evaluation of this expression yields a lower bound of $1.536 \ldots$.

\section{2-Dimensional Bin Packing}

Let $k \geq 1$ and let $L=L_{1 k} L_{0 k} L_{1(k-1)} \ldots L_{00}$ be a concatenation of $2(k+1)$ sublists $L_{g j}, g \in\{0,1\}, 0 \leq j \leq k$. Every sublists $L_{g j}$ contains $n$ equal sized items, which we 
will denote by $a_{g j}$. An item $a_{g j}$ has width $w\left(a_{g j}\right)$ and height $h\left(a_{g j}\right)$. The sizes are as follows $(0 \leq j \leq k)$ :

$$
\begin{array}{ll}
w\left(a_{1 j}\right)=\frac{1}{2}-(j+1) \varepsilon & h\left(a_{1 j}\right)=\frac{1}{m_{j}+1}+\varepsilon \\
w\left(a_{0 j}\right)=\frac{1}{2}+(j+1) \varepsilon & h\left(a_{0 j}\right)=\frac{1}{m_{j}+1}+\varepsilon
\end{array}
$$

We take $\varepsilon>0$ small enough to satisfy $\sum_{j=0}^{k} h\left(a_{1 j}\right) \leq 1$ and $w\left(a_{1 k}\right)>\frac{1}{3}$. So, $\varepsilon<$ $\min \left(\frac{1}{(k+1) m_{k+1}}, \frac{1}{6(k+1)}\right)$.

Then we can easily prove the following about the optimal packing of the partial list $L_{1 k} \ldots L_{g j}, g \in\{0,1\}, 0 \leq j \leq k$.

Lemma 3. If $n$ is a multiple of $2 m_{k}$, then
a) $\operatorname{OPT}\left(L_{1 k}\right) \leq \frac{n}{2 m_{k}}$
b) For every $0 \leq j \leq k: \operatorname{OPT}\left(L_{1 k} \ldots L_{0 j}\right) \leq \frac{n}{m_{j}}$
c) For every $0 \leq j \leq k-1: \mathrm{OPT}\left(L_{1 k} \ldots L_{1 j}\right) \leq \frac{n}{2}\left(\frac{1}{m_{j}}+\frac{1}{\mathrm{~m}_{j+1}}\right)$.

Proof:

Case a: It is easy to see that we can pack 2 items of $L_{1 k}$ side by side and that we can pack $m_{k}$ items of $L_{1 k}$ on top of each other. This means that $2 m_{k}$ items of $L_{1 k}$ can be packed together in a bin. So, $L_{1 k}$ can be packed in $\frac{n}{2 m_{k}}$ bins.

Case $b$ : If we pack $a_{1 p}$ and $a_{0 p}, j \leq p \leq k$, side by side and place $m_{j}$ pairs $\left(a_{1 p}, a_{0 p}\right)$, $j \leq p \leq k$, on top of each other, the total height in a bin will be

$$
\begin{aligned}
m_{j} \sum_{p=j}^{k}\left(\frac{1}{m_{p}+1}+\varepsilon\right) & =m_{j} \sum_{p=j}^{k}\left(\frac{1}{m_{p}}-\frac{1}{m_{p+1}}+\varepsilon\right) \\
& =1-m_{j}\left(\frac{1}{m_{k+1}}-(k-j+1) \varepsilon\right) \leq 1,
\end{aligned}
$$

because of $\varepsilon \leq \frac{1}{(k+1) m_{k+1}}$. So, $\frac{n}{m_{j}}$ bins are sufficient to pack this list.

Case c: With a strip of size $h$, we will mean an area of width 1 and height $h$. We can pack an item $a_{1 p}$ together with an item $a_{0 p}$ in a strip $S_{p}$ of size $\frac{1}{m_{p}+1}+\varepsilon, j+1 \leq$ $p \leq k$. Further, we can place two items $a_{1 j}$ together in a strip $S_{A}$ of size $\frac{1}{m_{j}+1}+\varepsilon$. 
If we pack $\frac{n}{2 m_{j}}$ bins with each $m_{j}$ strips $S_{A}$ and $m_{j}$ strips $S_{p}, j+1 \leq p \leq k$, and we pack $\frac{n}{2 m_{j+1}}$ bins with each $m_{j+1}$ strips $S_{p}, j+1 \leq p \leq k$, we get a feasible packing with $\frac{n}{2}\left(\frac{1}{m_{j}}+\frac{1}{m_{j+1}}\right)$ bins in total.

With a little more effort, one can also show that the inequalities in the this lemma hold with equality. However, this is not necessary for our technique to be applied. The optimal packing of the whole list $L$ is given in Fig. 1 .

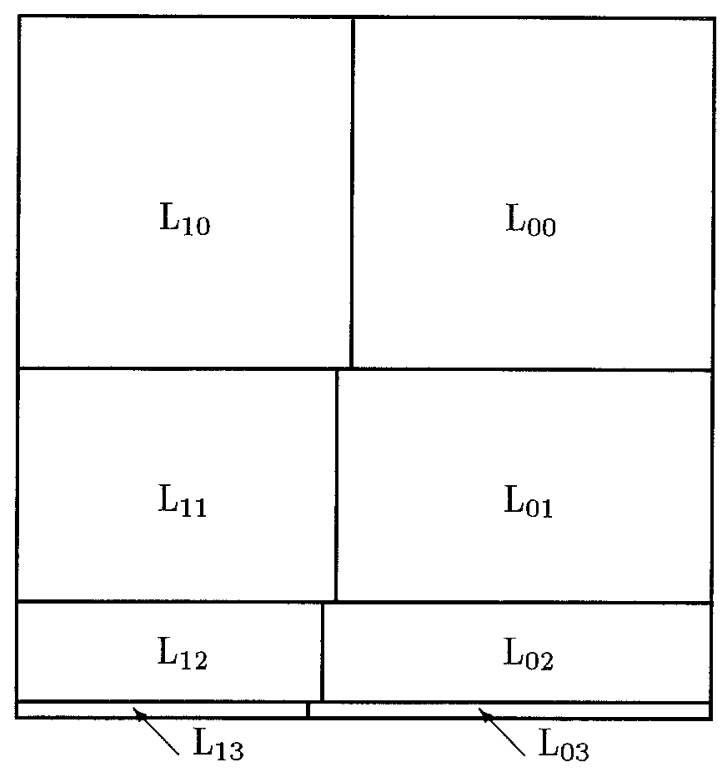

Figure 1. Optimal packing pattern for $k=3$

On the other hand, we can also find suitable weights that lead us to the following lemma.

Lemma 4. Let the weights $w_{g j}, g \in\{0,1\}, 0 \leq j \leq k$ be given by $w_{g j}=\frac{j+1}{m_{j}}$. Then we get for all $0 \leq p \leq k$ that
a) for every $\mathbf{t} \in T_{0 p} \sum_{j=0}^{p-1} t_{1 j} w_{1 j}+\sum_{j=0}^{p} t_{0 j} w_{0 j} \leq 2 p+1$
b) for every $\mathbf{t} \in T_{1 p} \sum_{j=0}^{p} t_{1 j} w_{1 j}+\sum_{j=0}^{p} t_{0 j} w_{0 j} \leq 2 p+2$. 
Proof:

First, we will deal with the case $p=0$. Since $w\left(a_{00}\right)>\frac{1}{2}$ and $h\left(a_{00}\right)>\frac{1}{2}$ it is immediately clear that a packing $\mathbf{t} \in T_{00}$ can contain only one item of $L_{00}$. Because of $w_{00}=1$ it follows that the condition of Case $a$ holds. Since $h\left(a_{00}\right)=h\left(a_{10}\right)>\frac{1}{2}$, a packing $\mathbf{t} \in T_{10}$ can contain at most 2 items that are placed side by side. So the weights $w_{10}=w_{00}=1$ satisfy the condition of Case $b$.

Now we can assume that $p \geq 1$. We will first introduce the notion of domination. We say that $m$ items $a_{g_{2} j_{2}}$ dominate one item $a_{g_{1} j_{1}}$ if

$$
\begin{aligned}
w\left(a_{g_{2} j_{2}}\right) & \leq w\left(a_{g_{1} j_{1}}\right) \\
m h\left(a_{g_{2} j_{2}}\right) & \leq h\left(a_{g_{1} j_{1}}\right) \\
m w_{g_{2} j_{2}} & \geq w_{g_{1} j_{1}}
\end{aligned}
$$

and at least one of these inequalities is strict. We denote this dominance by $m a_{g_{1} j_{2}}>a_{g_{1} j_{1}}$. So according to this definition we get: $a_{1 j}>a_{0 j}$ for all $0 \leq j \leq k$ and $m_{j} a_{1(j+1)} \succ a_{1 j}$ for all $0 \leq j \leq k-1$. We will now handle the Cases $a$ and $b$ separately:

Case a: We let $F_{0 p}(\mathbf{t})=\sum_{j=0}^{p-1} w_{1 j} t_{1 j}+\sum_{j=0}^{p} w_{0 p} t_{0 p}$. It suffices to prove that

$$
\max _{\mathbf{t} \in T_{0 p}} F_{0 p}(\mathbf{t}) \leq 2 p+1
$$

Because of the dominance rules we only need to consider items of $L_{0 p}$ and $L_{1(p-1)}$ in order to maximize $F_{0 p}(\mathbf{t})$. Since $w\left(a_{0 p}\right)+w\left(a_{1(p-1)}\right)>1$, a packing with maximum weight consists of a number of strips $S_{p}$ of size $\frac{1}{m_{p}+1}+\varepsilon$ with 1 item $a_{0 p}$ each, and of a number of strips $S_{p-1}$ of size $\frac{1}{m_{p-1}+1}+\varepsilon$ that contain 2 items $a_{1(p-1)}$ each. If we count the number of strips $S_{p}$ by $q_{p}$ and the number of strips $S_{p-1}$ by $q_{p-1}$, we get that $\max _{\mathbf{t} \in T_{0 p}} F_{0 p}(\mathbf{t})$ is equal to

$$
\max q_{p} \frac{p+1}{m_{p}}+2 q_{p-1} \frac{p}{m_{p-1}}
$$

under the condition that $q_{p}$ and $q_{p-1}$ satisfy

$$
\frac{q_{p}}{m_{p}+1}+\frac{q_{p-1}}{m_{p-1}+1}<1
$$

This maximization problem is solved by $q_{p}=q_{p-1}=m_{p-1}$ and has an optimal solution of $2 p+\frac{p+1}{m_{p-1}+1}$ which is less than or equal to $2 p+1$.

Case b: Because of the dominance rules we only have to consider items of $L_{1 p}$. At most 2 items $a_{1 p}$ can be packed beside each other, and at most $m_{p}$ items $a_{1 p}$ can be placed on top of each other. This means that the maximum weight in a packing is equal to $2 m_{p} \frac{p+1}{m_{p}}=2 p+2$. 
This leads us to the following theorem.

Theorem 5. Every on-line algorithm for the 2-dimensional bin packing problem has an asymptotic worst case ratio of at least

$$
\lim _{k \rightarrow \infty} \frac{2 \sum_{j=0}^{k} \frac{j+1}{m_{j}}}{\sum_{j=0}^{k} \frac{1}{m_{j}}+\frac{1}{2}\left[\frac{1}{m_{k}}+\sum_{j=0}^{k-1}\left(\frac{1}{m_{j}}+\frac{1}{m_{j+1}}\right)\right]} .
$$

Proof:

This follows directly from Lemma 3, Lemma 4 and Theorem 1.

Evaluation of this formula gives us a lower bound of $1.802 \ldots$

\section{3-Dimensional Bin Packing}

Given $k \geq 1$, let $L=L_{11 k} L_{01 k} L_{10 k} L_{00 k} L_{11(k-1)} \ldots L_{000}$ be a concatenation of $4(k+1)$ sublists $L_{f g j}, f, g \in\{0,1\}, 0 \leq j \leq k$, of 3 -dimensional items. Each sublist $L_{f g j}$ contains $n$ items of the same size, which we will denote by $a_{f g j}$. An item $a_{f g j}$ has width $w\left(a_{f g j}\right)$, height $h\left(a_{f g j}\right)$ and depth $d\left(a_{f g j}\right)$ as follows $(0 \leq j \leq k)$ :

$$
\begin{array}{lll}
w\left(a_{11 j}\right)=\frac{1}{2}-(2 j+2) \varepsilon & h\left(a_{11 j}\right)=\frac{1}{2}-(j+1) \varepsilon & d\left(a_{11 j}\right)=\frac{1}{m_{j}+1}+\varepsilon \\
w\left(a_{01 j}\right)=\frac{1}{2}+(2 j+2) \varepsilon & h\left(a_{01 j}\right)=\frac{1}{2}-(j+1) \varepsilon & d\left(a_{01 j}\right)=\frac{1}{m_{j}+1}+\varepsilon \\
w\left(a_{10 j}\right)=\frac{1}{2}-(2 j+1) \varepsilon & h\left(a_{10 j}\right)=\frac{1}{2}+(j+1) \varepsilon & d\left(a_{10 j}\right)=\frac{1}{m_{j}+1}+\varepsilon \\
w\left(a_{00 j}\right)=\frac{1}{2}+(2 j+1) \varepsilon & h\left(a_{00 j}\right)=\frac{1}{2}+(j+1) \varepsilon & d\left(a_{00 j}\right)=\frac{1}{m_{j}+1}+\varepsilon
\end{array}
$$

We take $\varepsilon \leq \frac{1}{2(k+1) m_{k+1}}$. This guarantees that at most 2 items can be placed side by side or on top of each other and also that the items $a_{f g 0}, a_{f g 1}, \ldots, a_{f g k}$ can be placed behind each other for every $f, g \in\{0,1\}$. The optimal packing of $L$ takes $n$ bins and is given in Fig. 2.

In the remainder we will mean with a slice of size $s$ an area of width and height equal to 1 and depth equal to $s$. So, the items $a_{11 j}, a_{01 j}, a_{10_{j}}$ and $a_{00 j}$ can be packed together in a slice of size $\frac{1}{m_{j}+1}+\varepsilon$.

We can prove the following upper bounds on the optimal solution of the concatenated lists $L_{11 k} \ldots L_{f g j}$ for every $f, g \in\{0,1\}$ and $0 \leq j \leq k$. 


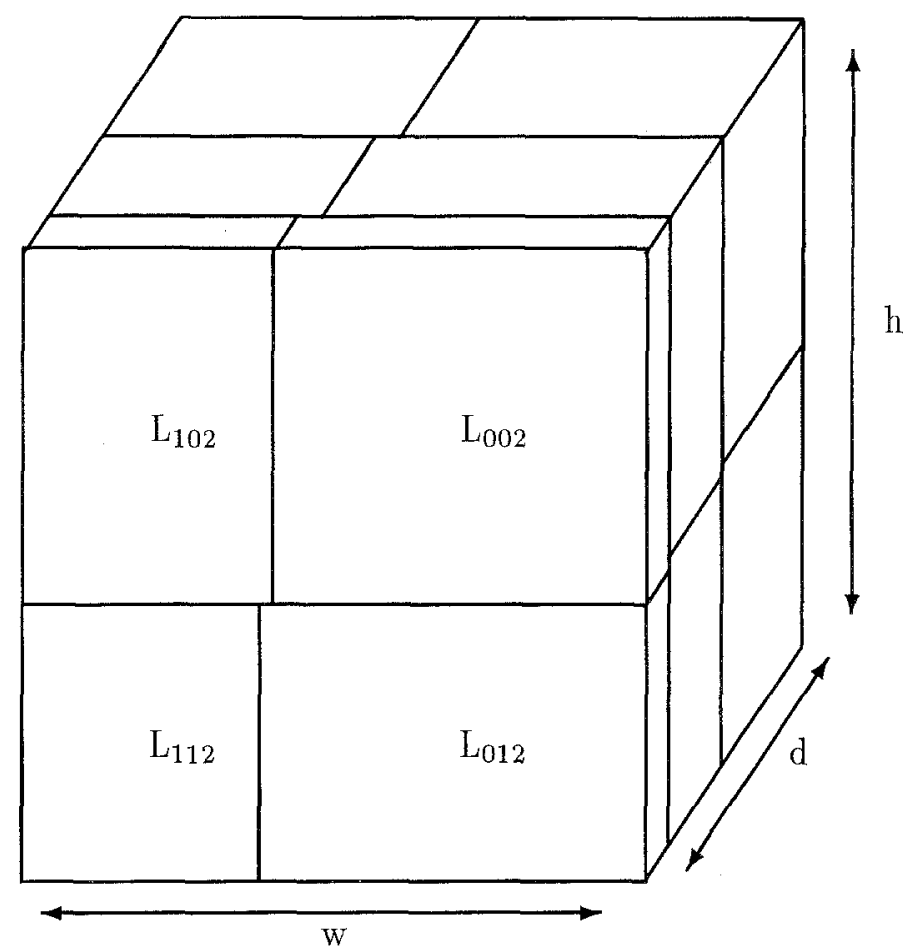

Figure 2. Optimal packing pattern for $k=2$

Lemma 6. Let $n$ be a multiple of $4 m_{k}$, then
a) $\operatorname{OPT}\left(L_{11 k}\right) \leq \frac{n}{4 m_{k}}$,
b) $\operatorname{OPT}\left(L_{11 k} L_{01 k}\right) \leq \frac{2 n}{4 m_{k}}$,
c) $\operatorname{OPT}\left(L_{11 k} L_{01 k} L_{10 k}\right) \leq \frac{3 m}{4 m_{k}}$
d) For every $0 \leq j \leq k$ : OPT $\left(L_{11 k} \ldots L_{00 j}\right) \leq \frac{n}{m_{j}}$,
e) For every $0 \leq j \leq k-1: \operatorname{OPT}\left(L_{11 k} \ldots L_{11 j}\right) \leq \frac{n}{4}\left(\frac{1}{m_{j}}+\frac{3}{m_{j+1}}\right)$,
f) For every $0 \leq j \leq k-1: \operatorname{OPT}\left(L_{1 k} \ldots L_{01 j}\right) \leq \frac{n}{4}\left(\frac{2}{m_{j}}+\frac{2}{m_{j+1}}\right)$,
g) For every $0 \leq j \leq k-1$ : $\operatorname{OPT}\left(L_{11 k} \ldots L_{10 j}\right) \leq \frac{n}{4}\left(\frac{3}{m_{j}}+\frac{1}{m_{j+1}}\right)$.

Proof:

We will prove this lemma by giving feasible packings that use the indicated number of bins. 
Case a: We will pack 2 items side by side, 2 items on top of each other and $m_{k}$ items of $L_{k}$ behind each other in a bin. Because of $w\left(a_{11 k}\right)<\frac{1}{2}, h\left(a_{11 k}\right)<\frac{1}{2}$ and $d\left(a_{11 k}\right)<$ $\frac{1}{m_{k}}$, this packing will be feasible. It packs $4 m_{k}$ items together in a bin and since $n$ is a multiple of $4 m_{k}$, we need $\frac{n}{4 m_{k}}$ bins.

Case b: We can pack an item of $L_{11 k}$ and an item of $L_{01 k}$ side by side because of $w\left(a_{11 k}\right)+w\left(a_{01 k}\right)=1$. They have the same height and the same depth and by repeating this pattern 2 times in height and $m_{k}$ times in depth, we get a feasible packing. Every bin contains $2 m_{k}$ items of both $L_{11 k}$ and $L_{01 k}$ and because $n$ is a multiple of $2 m_{k}$, we will need exactly $\frac{2 n}{4 m_{k}}$ bins.

Case c: We can pack 2 items of $L_{10 k}$ together with 1 item of $L_{11 k}$ and 1 item of $L_{01 k}$ in a slice $S_{C}$ of size $\frac{1}{m_{k}+1}+\varepsilon$. We can also pack 2 items of $L_{11 k}$ together with 2 items of $L_{01 k}$ in a slice $S_{B}$ of size $\frac{1}{m_{k}+1}+\varepsilon$ (as in Case $b$ ). If we fill $\frac{n}{2 m_{k}}$ bins each with $m_{k}$ slices $S_{C}$ and we fill $\frac{n}{4 m_{k}}$ bins with $m_{k}$ slices $S_{B}$, then we have a feasible packing with $\frac{3 n}{4 m_{k}}$ bins.

Case d: We can pack 1 item of every list $L_{11 p}, L_{01 p}, L_{10_{p}}$ and $L_{00_{p}}$ together in a slice $S_{p}$ of size $\frac{1}{m_{p}+1}+\varepsilon, j \leq p \leq k$. If we put $m_{j}$ slices of every $S_{p}, j \leq p \leq k$ together, then the total depth will be equal to

$$
\begin{aligned}
m_{j} \sum_{p=j}^{k}\left(\frac{1}{m_{p}+1}+\varepsilon\right) & =m_{j} \sum_{p=j}^{k}\left(\frac{1}{m_{p}}-\frac{1}{m_{p+1}}+\varepsilon\right) \\
& =1-m_{j}\left(\frac{1}{m_{k+1}}-(k-j+1) \varepsilon\right)<1
\end{aligned}
$$

because of $\varepsilon \leq \frac{1}{2(k+1) m_{k+1}}$. This means that the packing of this bin is feasible and we need $\frac{n}{m_{j}}$ bins in total.

Case e: We consider slices $S_{A}$ of size $\frac{1}{m_{j}+1}+\varepsilon$ that contain four items of $L_{11 j}$. Further we have slices $S_{p}$ of size $\frac{1}{m_{p}+1}+\varepsilon, j+1 \leq p \leq k$, that contain one item of every list $L_{11 p}, L_{01 p}, L_{10_{p}}$ and $L_{00 p}$. We pack $\frac{n}{4 m_{j}}$ bins with $m_{j}$ slices $S_{A}$ and $m_{j}$ 
slices $S_{p}, j+1 \leq p \leq k$, and we pack $\frac{3 n}{4 m_{j+1}}$ bins with $m_{j+1}$ slices $S_{p}, j+1 \leq p \leq k$. It is easy to verify that this gives a feasible packing. Adding up gives $\frac{n}{4}\left(\frac{1}{m_{j}}+\frac{3}{m_{j+1}}\right)$ bins.

Case f: We consider slices $S_{B}$ of size $\frac{1}{m_{j}+1}+\varepsilon$ that contain 2 items of $L_{11 j}$ and 2 items of $L_{01 j}$. Further we have slices $S_{p}, j+1 \leq p \leq k$, as in Case $e$. We pack $\frac{n}{2 m_{j}}$ bins with $m_{j}$ slices $S_{B}$ and $m_{j}$ slices $S_{p}, j+1 \leq p \leq k$, and we pack $\frac{n}{2 m_{j}+1}$ bins with $m_{j+1}$ slices $S_{p}, j+1 \leq p \leq k$. This packing is feasible and the result follows.

Case g: We consider slices $S_{C}$ of size $\frac{1}{m_{j}+1}+\varepsilon$ that contain 1 item of $L_{11 j}, 1$ item of $L_{01 j}$ and 2 items of $L_{10 j}$. Further we have slices $S_{p}, j+1 \leq p \leq k$, as in Case $e$ and slices $S_{B}$ as in Case $f$. We pack $\frac{n}{2 m_{j}}$ bins with $m_{j}$ slices $S_{C}$ an $m_{j}$ slices $S_{p}$, $j+1 \leq p \leq k$, we pack $\frac{n}{4 m_{j}}$ bins with $m_{j}$ slices $S_{B}$ and $m_{j}$ slices $S_{p}, j+1 \leq p \leq k$, and we pack $\frac{n}{4 m_{j+1}}$ bins with $m_{j+1}$ slices $S_{p}, j+1 \leq p \leq k$. This packing is feasible, so the desired result follows.

The next lemma states the results about the packings $t \in T_{f g j}$.

Lemma 7. Let $w_{f g j}=\frac{j+1}{m_{j}}, f, g \in\{0,1\}, 0 \leq j \leq k$. Then for all $0 \leq p \leq k$ we have

a) For every $\mathbf{t} \in T_{00 p}$

$$
\sum_{f=0}^{1} \sum_{g=0}^{1} \sum_{j=0}^{p-1} w_{f g j} t_{f g j}+w_{00 p} t_{00 p} \leq 4 p+1
$$

b) For every $\mathbf{t} \in T_{10 p}$

$$
\sum_{f=0}^{1} \sum_{g=0}^{q} \sum_{j=0}^{p-1} w_{f g j} t_{f g j}+w_{10 p} t_{10 p}+w_{00_{p}} t_{00_{p}} \leq 4 p+2
$$

c) For every $\mathbf{t} \in T_{01 p}$

$$
\sum_{f=0}^{1} \sum_{g=0}^{1} \sum_{j=0}^{p-1} w_{f g j} t_{f g j}+w_{01 p} t_{01 p}+w_{10 p} t_{10 p}+w_{00 p} t_{00 p} \leq 4 p+3
$$

d) For every $\mathbf{t} \in T_{11 p}$

$$
\sum_{j=0}^{1} \sum_{g=0}^{1} \sum_{j=0}^{p} w_{f g j} t_{f g j} \leq 4 p+4
$$


Proof:

We will first deal with the case $p=0$. Since $w_{f g 0}=1, f, g \in\{0,1\}$, we have to show that if $\mathrm{t} \in T_{000}$ (resp. $T_{100}, T_{101}$ or $T_{110}$ ) that such a packing $\mathrm{t}$ can contain at most 1 (resp. 2,3 or 4 ) item(s). We will leave this to the reader as an easy exercise.

From now on we will assume $p \geq 1$. We will first introduce the notion of domination. We say that $m$ items $a_{f_{2} g_{2} j_{2}}$. dominate one item $a_{f_{1} g_{1} j_{1}}$ if

$$
\begin{aligned}
w\left(a_{f_{2} g_{2} j_{2}}\right) & \leq w\left(a_{f_{1} g_{1} j_{1}}\right) \\
h\left(a_{f_{2} g_{2} j_{2}}\right) & \leq h\left(a_{f_{1} g_{1} j_{1}}\right) \\
m d\left(a_{f_{2} g_{2} j_{2}}\right) & \leq d\left(a_{f_{1} g_{1} j_{1}}\right) \\
m w_{f_{2} g_{2} j_{2}} & \geq w_{f_{1} g_{1} j_{1}}
\end{aligned}
$$

and at least one of these inequalities is strict. We denote this dominance by $m a_{f_{2} g_{2} j_{2}}>a_{f_{1} g_{1} j_{1}}$

An other useful tool that we will use is a 3-dimensional coordinate system. We can think of a bin as being placed in a 3-dimensional coordinate system such that it covers the area $[0,1] \times[0,1] \times[0,1]$. We let the width of the bin correspond with the first dimension, the height with the second dimension and the depth with the third dimension. This coordinate system allows us to speak about planes and lines intersecting the bin, which we will use for the proof of the Cases $a, b$ and $c$.

Case a: We let $F_{00 p}(\mathbf{t})=\sum_{f=0}^{1} \sum_{g=0}^{1} \sum_{j=0}^{p-1} w_{f g j} t_{f g j}+w_{00 p} t_{00 p}$. It suffices to prove that

$$
\max _{\mathbf{t} \in T_{00 p}} F_{00 p}(\mathbf{t}) \leq 4 p+1 .
$$

Because $a_{11 j} \succ a_{10 j}, \quad a_{11 j} \succ a_{01 j}, \quad a_{11 j} \succ a_{00 j}$ for every $0 \leq j \leq p-1$ and $m_{j} a_{11(j+1)} \succ a_{11 j}$ for every $0 \leq j \leq p-1$, we only have to consider items of $L_{00 p}$ and $L_{11(p-1)}$ in order to maximize $F_{00 p}(\mathbf{t})$.

If we intersect the bin with a plane $P$ given by $x_{3}=c, 0 \leq c \leq 1$, we will encounter at most 4 items. Because 4 items of $L_{11(p-1)}$ can be packed together in a slice of size $\frac{1}{m_{p-1}+1}+\varepsilon$, we can encounter 4 items of $L_{11(p-1)}$. Whenever $P$ intersects an item of $L_{00 p}$, that will be the only item it intersects, because of $w\left(a_{00 p}\right)>\frac{1}{2}, h\left(a_{00 p}\right)>\frac{1}{2}$, $w\left(a_{00 p}\right)+w\left(a_{11(p-1)}\right)>1$ and $h\left(a_{0 o p}\right)+h\left(a_{11(p-1)}\right)>1$.

When we intersect the bin with a line $l$ given by $x_{1}=a, x_{2}=b, 0 \leq a, b \leq 1$, we count the number of items of $L_{00 p}$ that we intersect by $q_{p}$ and the number of items of $L_{11(p-1)}$ by $q_{p-1}$. Of course $q_{p} * d\left(a_{00 p}\right)+q_{p-1} * d\left(a_{11(p-1)}\right) \leq 1$ must be satisfied. Because this must be satisfied for every line $l$, we can bound $\max _{\mathrm{t} \in T_{0 o_{p}}} F_{00 p}(\mathbf{t})$ by

$$
\max q_{p} \frac{p+1}{m_{p}}+4 q_{p-1} \frac{p}{m_{p-1}}
$$


under the condition that $q_{p}$ and $q_{p-1}$ satisfy

$$
\frac{q_{p}}{m_{p}+1}+\frac{q_{p-1}}{m_{p-1}+1}<1
$$

This maximization problem is solved by $q_{p}=q_{p-1}=m_{p-1}$ and has an optimal solution of $4 p+\frac{p+1}{m_{p-1}+1}$ which is less than or equal to $4 p+1$.

Case b: We let $F_{10 p}(\mathbf{t})=\sum_{f=0}^{1} \sum_{g=0}^{1} \sum_{j=0}^{p-1} w_{f g j} t_{f g j}+w_{00 p} t_{00 p}+w_{10 p} t_{10 p}$. It suffices to prove that

$$
\max _{\mathbf{t} \in T_{0_{p}}} F_{10 p}(\mathbf{t}) \leq 4 p+2 .
$$

After applying some evident dominance rules, only items of $L_{10 p}$ and $L_{11(p-1)}$ remain to maximize $F_{10 p}(\mathbf{t})$. Because $w\left(a_{10 p}\right)<\frac{1}{2}$ and $w\left(a_{11(p-1)}\right)<\frac{1}{2}$ we can split the problem of maximizing the value of the items in the bin into 2 equivalent problems: maximize the value of the items in the left (resp. right) half of the bin. We will work with the left side of the bin. Let the half-plane $H$ be given by $x_{3}=c$ and $x_{1} \leq \frac{1}{2}$. Because of the height of $a_{11(p-1)}$ and $a_{10 p}, H$ can intersect at most 2 items of $a_{11(p-1)}$ or 1 item of $a_{10 p}$. With $q_{p}$ (resp. $q_{p-1}$ ) we count the number of items $a_{10 p}$ (resp. $a_{1(p-1)}$ ) that we encounter on the line $l$ given by $x_{1}=a$ and $x_{2}=b, 0 \leq a \leq \frac{1}{2}$, $0 \leq b \leq 1$. We can bound the value of the items in the left half of the bin by

$$
\max 2 q_{p-1} \frac{p}{m_{p-1}}+q_{p} \frac{p+1}{m_{p}}
$$

under the condition that $q_{p}$ and $q_{p-1}$ satisfy

$$
\frac{q_{p-1}}{m_{p-1}+1}+\frac{q_{p}}{m_{p}+1}<1
$$

This is solved by $q_{p}=q_{p-1}=\frac{1}{m_{p-1}}$ and yields a value of $2 p+\frac{p+1}{m_{p-1}+1}$. Since the right half of the bin yields the same outcome and since $2\left(2 p+\frac{p+1}{m_{p-1}+1}\right) \leq 4 p+2$ this completes the proof of this case.

Case $\quad c:$ We let $F_{01 p}(\mathbf{t})=\sum_{f=0}^{1} \sum_{g=0}^{1} \sum_{j=0}^{p-1} w_{f g j} t_{f g j}+w_{00 p} t_{00 p}+w_{10 p} t_{10 p}+$ $w_{01 p} t_{01 p}$. It suffices to prove that

$$
\max _{\mathbf{t} \in T_{01 p}} F_{01 p}(\mathbf{t}) \leq 4 p+3
$$

Applying the dominance rules leaves us with items of $L_{11(p-1)}, L_{01 p}$ and $L_{10 p}$ to maximize $F_{01 p}(\mathbf{t})$. When we intersect the bin with a plane $P$ given by $x_{3}=c$, $0 \leq c \leq 1$, we may encounter 4 items $a_{11(p-1)}$. However, if $P$ intersects at least 1 item $a_{10 p}$ or $a_{01 p}$, then it can intersect at most 3 items $\left(2 \times a_{10 p}\right.$ and $1 \times a_{01 p}$, $2 \times a_{11(p-1)}$ and $1 \times a_{01 p}$, or $2 \times a_{11(p-1)}$ and $1 \times a_{10 p}$; however, only the first possibility is relevant for maximizing $\left.F_{01 p}(t)\right)$. When we intersect the bin with a line $l$ given by $x_{1}=a, x_{2}=b, 0 \leq a, b \leq 1$, we count the number of items of $L_{01 p}$ and 
$L_{10 p}$ that we intersect by $q_{p}$ and the number of items of $L_{11(p-1)}$ by $q_{p-1}$. Of course $q_{p} * d\left(a_{01 p}\right)+q_{p-1} * d\left(a_{11(p-1)}\right) \leq 1$ must be satisfied (note that $\left.d\left(a_{01 p}\right)=d\left(a_{10 p}\right)\right)$. Because this must be satisfied for every line $l$, we can bound $\max _{\mathbf{t} \in T_{01 p}} F_{01 p}(\mathbf{t})$ by

$$
\max 4 q_{p-1} \frac{p}{m_{p-1}}+3 q_{p} \frac{p+1}{m_{p}}
$$

under the condition that $q_{p}$ and $q_{p-1}$ satisfy

$$
\frac{q_{p-1}}{m_{p-1}+1}+\frac{q_{p}}{m_{p}+1}<1
$$

This is solved by $q_{p}=q_{p-1}=m_{p-1}$, that yields a value of $4 p+3 \frac{p+1}{m_{p-1}+1}$ which is less than or equal to $4 p+3$.

Case d: We let $F_{11 p}(\mathbf{t})=\sum_{f=0}^{l} \sum_{g=0}^{1} \sum_{j=0}^{p} w_{f g j} t_{f g j}$. We need to prove that

$$
\max _{\mathbf{t} \in T_{11 p}} F_{11 p}(\mathbf{t}) \leq 4 p+4 .
$$

Because of the dominance rules, we only have to consider items of $L_{11 p}$ in order to maximize $F_{11 p}(\mathbf{t})$.

But only $4 m_{p}$ items of $L_{11 p}$ can be packed together into a bin, so $\max _{\mathbf{t} \in T_{11 p}} F_{11 p}(\mathbf{t})$ is equal to $4 m_{p} \frac{p+1}{m_{p}}=4 p+4$, which verifies the last part of this lemma.

Theorem 8. Every on-line algorithm for the 3-dimensional bin packing problem has an asymptotic worst case ratio of at least

$$
\lim _{k \rightarrow \infty} \frac{4 \sum_{j=0}^{k} \frac{j+1}{\sum_{j=0}} \frac{1}{m_{j}}+\frac{3}{2}\left[\frac{1}{m_{k}}+\sum_{j=0}^{k-1}\left(\frac{1}{m_{j}}+\frac{1}{m_{j+1}}\right)\right]}{.}
$$

Proof:

This follows directly from Lemma 6, Lemma 7 and Theorem 1.

Some calculation gives us a lower bound of $1.974 \ldots$.

\section{Conclusion}

It seems that a possible generalization derives lower bounds for the $d$-dimensional bin packing problem for $d>3$. In $d-1$ dimensions the items will have size of about $\frac{1}{2}$, and in 1 dimension the items will be of size $\frac{1}{m_{j}+1}+\varepsilon$. Note that this covers dimension 1, 2 and 3 as well. We will state our ideas without proof.

Conjecture 9. Every on-line algorithm for the d-dimensional bin packing problem has an asymptotic worst case ratio of at least 


$$
\lim _{k \rightarrow \infty} \frac{2^{d-1} \sum_{j=0}^{k} \frac{j+1}{m_{j}}}{\sum_{j=0}^{k} \frac{1}{m_{j}}+\frac{2^{(d-1)}-1}{2}\left[\frac{1}{m_{k}}+\sum_{j=0}^{k-1}\left(\frac{1}{m_{j}}+\frac{1}{m_{j+1}}\right)\right]}
$$

If we let the dimension $d$ grow to infinity, the lower bound converges to $2.181 \ldots$. So, unlike the asymptotic worst case ratio of the best on-line algorithm this lower bound does not grow exponentially with the dimension, but it remains under a constant. It may be an interesting question whether it is possible to find a lower bound that depends on $d$ at least logarithmically, either by refining the construction of the list or by a better proof technique. We summarized the values of the lower bounds in Table 1 .

Table 1. Lower bounds for the $d$-dimensional bin packing problem

\begin{tabular}{|c|c|}
\hline$d$ & Lower bound \\
\hline 1 & $1.536 \ldots$ \\
2 & $1.802 \ldots$ \\
3 & $1.974 \ldots$ \\
$\infty$ & $2.181 \ldots(?)$ \\
\hline
\end{tabular}

As we already discussed in Section 2, the quality of the lower bound depends on the gap in the inequality (2) for packings produced by an algorithm that minimizes $r=\max _{j}\left\{\lim _{n \rightarrow \infty} r_{j}(n)\right\}$. Van Vliet [9] computes such an optimal algorithm for the list $L$ that we used in Section 3 for the 1 -dimensional bin packing problem, by means of a linear programming formulation. Indeed, he shows that there exists a gap in (2) for some of the resulting packings. This linear programming formulation also gives an improved lower bound of $1.540 \ldots$ Given our construction of $L$, this is the best possible. It seems that, at a cost of much more work, it is possible to extend this linear programming approach to dimensions 2 and 3 as well.

\section{References}

[1] Csirik, J., Van Vliet, A.: An on-line algorithm for multidimensional bin packing. Oper. Res. Lett, (to appear).

[2] Galambos, G.: A 1.6 lower bound for the two-dimensional on-line rectangle bin packing. Acta Cybernetica 10, 21-24 (1991).

[3] Galambos, G., Frenk, J. B. G.: A simple proof of Liang's lower bound for on-line bin packing and the extension to the parametric case. Discr. Appl. Math. 41, 173-178 (1993)

[4] Galambos, G., Kellerer, H., Woeginger G.: A lower bound for on-line vector-packing algorithms. Report no. 182, Institute für Mathematik, Technische Universität Graz (1991).

[5] Garey, M. R., Johnson, D. S.: Computers and intractability: a guide to the theory of NP-completeness. San Francisco: W. H. Freeman, 1979.

[6] Li, K., Cheng, K. H.: A generalized harmonic algorithm for on-line multi-dimensional bin packing. Unpublished manuscript, 1990.

[7] Liang, F. M.: A lower bound for on-line bin packing. Inf. Proc. Lett. 10, 76-79 (1980). 
[8] Richey, M. B.: Improved bounds for refined harmonic bin packing. Unpublished manuscript, 1990.

[9] Van Vliet, A.: An improved lower bound for on-line bin packing algorithms. Inf. Proc. Lett. 43, 277-284 (1992).

Dr. G. Galambos

Department of Computer Science

JGYTF, P.O. Box 396

H-6720 Szeged

Hungary
Dr. A. van Vliet

Econometric Institute

Erasmus University Rotterdam

P.O. Box 1738

3000 DR Rotterdam

The Netherlands 